\title{
Cysticercosis and cerebrovascular disease: a review
}

\author{
Oscar H Del Brutto
}

\begin{abstract}
Ischaemic cerebrovascular disease is a relatively common but under-recognised complication of neurocysticercosis. It is usually caused by inflammatory occlusion of the arteries at the base of the brain secondary to cysticercotic arachnoiditis. In most cases, the involved vessels are of small diameter and the neurological picture is limited to a lacunar syndrome secondary to a small cerebral infarct. However, large infarcts related to the occlusion of the middle cerebral artery or even the internal carotid artery have also been reported in this setting. CT and CSF examination usually support the causeand-effect relationship between neurocysticercosis and the cerebral infarct by showing abnormalities compatible with cysticercotic arachnoiditis. An accurate diagnosis of this condition is important since early treatment with steroids is advised to ameliorate the subarachnoid inflammatory reaction which may cause recurrent cerebral infarcts.
\end{abstract}

Cysticercosis is the most frequent parasitic disease of the CNS, and one of the most pleomorphic of neurological disorders. ${ }^{15} \mathrm{Clin}$ ical manifestations of neurocysticercosis (NCC) are nonspecific and largely depend on the number and topography of lesions and the host immune response to the parasite. ${ }^{143039}$ NCC is usually manifested by seizures, progressive intellectual deterioration, and signs and symptoms of increased intracranial pressure. ${ }^{41}$ In addition, a variety of focal neurological signs occur in many patients. While such deficits are usually related to parenchymal brain cysticerci, in an important number of patients they occur as the result of a cerebrovascular event. ${ }^{273845}$

Cerebrovascular complications of NCC were first described in the nineteenth century in a patient with cysticercotic meningitis who had angiitis of small intracranial vessels. ${ }^{29}$ Since then, several authors have recognised the association between NCC and cerebrovascular disease. ${ }^{21120283348}$ Most of these reports have focused on the histopathological and angiographic aspects of cysticercotic angiitis, but little emphasis has been placed on the clinical manifestations of this condition or in the therapeutic approach. In recent years, several patients with brain infarcts due to NCC have been reported, ${ }^{5-72634}$ nevertheless, stroke is an under-recognised complication of NCC, and authoritative textbooks of neurology ${ }^{138^{\circ}}$ do not consider this parasitic disease in the differential diagnosis of cerebral infarcts. This paper presents a review of the literature on this subject, and discusses the pathophysiology of cysticercosis induced cerebrovascular disease.

\section{Lacunar infarction}

This is the most common cerebrovascular complication of NCC. ${ }^{15}$ Cysticercosis-induced lacunar infarcts are usually located in the territory of the lenticulostriate branches of the anterior or middle cerebral artery, ${ }^{20}$ and result from occlusive endarteritis secondary to the intense inflammatory reaction within the subarachnoid space that is triggered by meningeal cysticerci. Such inflammatory reaction is composed of hyalinised parasitic membranes, collagen fibres, lymphocytes, plasma cells, eosinophils, and multinucleated giant cells. ${ }^{19}$ These changes cause abnormal thickening of the leptomeninges at the base of the skull with subsequent entrapment and occlusion of blood vessels around the circle of Willis.

Small cerebral infarcts due to NCC have been associated with lacunar syndromes which are indistinguishable from those caused by atherosclerosis. $^{5-7}$ These patients may present with an ataxic hemiparesis, pure motor hemiparesis, or sensorimotor stroke, due to a lacune that is usually located in the posterior limb of the internal capsule or the corona radiata. CT provides clues to the aetiology of the infarct as it shows associated abnormalities in most cases. In a series of seven patients with a lacunar syndrome due to NCC, we found CT evidence of NCC in six cases: four patients had a suprasellar cysticercus ipsilateral to the infarct, one had a large meningeal cyst in the vicinity of the infarct, and the other patient had hydrocephalus due to diffuse arachnoiditis. CSF analysis is of paramount importance in the evaluation of patients with suspected cysticercotic angiitis, as it shows a mild pleocytosis and elevated protein contents in most cases. In addition, immunological reactions (ELISA and complement fixation test) for the detection of anticysticercal antibodies are almost always positive in CSF due to active meningeal disease. ${ }^{35}$ Cerebral angiography may be completely normal because the involved vessels are too small to be imaged, or may show segmental narrowing of major intracranial vessels when the process of angiitis is more extensive. ${ }^{6}$

\section{Large infarctions}

Cerebral infarcts involving the deep and superficial territory of a major intracranial artery have occasionally been reported in patients 
with cysticercotic angiitis. A search of the literature reveals only five well-documented cases. $^{263447}$ These patients presented with profound neurological deficits secondary to an infarct that involved the basal ganglia, the cerebral cortex, or both. They had a protracted course and most of them died as the result of either the cerebral infarct or the associated arachnoiditis. CT was available in three of these patients and showed a large meningeal cysticercus adjacent to the brain infarct in every case. Angiography showed occlusion of the supraclinoid segment of the internal carotid artery in two patients, and occlusion of the middle cerebral artery in the remaining three. Postmortem examination of the brain of patients dying with this disorder showed interesting findings. Besides occlusive endarteritis, some blood vessels of large diameter showed occlusion of their lumens by atheroma-like deposits that resulted from disruption of the endothelium secondary to the invasion of the vessel wall by a severe inflammatory reaction induced by meningeal cysticerci. ${ }^{34}$

\section{Progressive midbrain syndrome}

This is one of the most devastating complications of NCC and has been associated with a mortality rate of $85 \% .^{50}$ Those patients usually have a history of shunted hydrocephalus due to diffuse cysticercotic arachnoiditis. In a typical case, the patient is readmitted a few months after the shunt was placed because of progressive neurological deterioration characterised by somnolence, paraparesis, impaired vertical gaze, fixed and dilated pupils, and urinary incontinence. CT shows normal-sized lateral ventricles and an ill-defined hypodense zone in the midbrain tegmentum and medial thalamus. CSF analysis shows marked pleocytosis and increased protein contents, with positive immunological reactions to cysticercosis. With the aid of MRI, it has been possible to visualise multiple areas of ischaemia in the midbrain and thalamus which are the result of the occlusion of paramedian thalamopeduncular branches of the mesencephalic artery. Such branches are prone to develop inflammatory occlusion as the interpeduncular and prepontine cisterns are where the gelatinous exudate associated with cysticercotic arachnoiditis is more intense. ${ }^{19}$ The prognosis of these patients is bad, and they usually die after a protracted course. ${ }^{46}$

\section{Transient ischaemic attacks}

There have been scarce reports of patients with NCC who complain of brief episodes of neurological deficit resembling transient ischaemic attacks. The first description of this association was made by Bickerstaff ${ }^{9}$ under the heading of "Transient loss of function". While lacking pathological or angiographic correlation, the author considered that such episodes were vascular in nature. McCormick et $\mathrm{al}^{26}$ reported a patient who experienced transient episodes of hemiparesis related to the upright posture which was resolved in the supine position. This patient had a large cysticercus around the internal carotid artery that occluded its lumen and caused positional cerebral ischaemia. On the other hand, we have recently described a patient with transient episodes of hemiparesis due to a large parenchymal brain cysticercus. ${ }^{16}$ Data from these reports suggest that NCC may cause transient neurological dysfunction resembling transient ischaemic attacks. The pathogenesis of this condition, however, is not uniform. It may be of vascular origin or may be related to parenchymal brain cysts in a similar way to that described in patients with cerebral tumours. ${ }^{36}$

\section{Discussion}

As shown in this review, several clinical reports have implied a link between NCC and ischaemic cerebrovascular disease. This association has been confirmed from data of large series showing that approximately $3 \%$ of patients with NCC develop a cerebral infarct, ${ }^{273845}$ and that $2.5 \%$ of patients with cerebral infarcts have NCC. ${ }^{4}$ The proportion of patients with cerebral infarcts due to NCC, however, varies according to the underlying prevalence of cysticercosis in a given population. In Mexico, cysticercosis is the second most frequent cause of non-atherosclerotic cerebral infarct. ${ }^{4}$ Such incidence could be representative of several other developing countries and industrialised nations with a high immigrant population from areas where this parasitic disease is endemic. ${ }^{1018212331}$

In endemic areas for cysticercosis, a patient may have NCC and a cerebral infarct from unrelated reasons. The diagnosis therefore of cysticercosis-induced cerebral infarct should be established only in patients who meet rigid inclusion criteria. These patients usually are younger than 40 years of age and have no risk factors for cerebrovascular disease. The causeand-effect relationship between NCC and a cerebral infarct must be supported by $\mathrm{CT}$ evidence of a meningeal cyst adjacent to the infarct or by CSF findings compatible with active arachnoiditis.

Many infectious diseases of the CNS have been associated with cerebrovascular disease, 22425374049 such as, the parallelism between the inflammatory arteriopathy caused by NCC and that caused by tuberculous meningitis. In both conditions, the arteries at the base of the brain are surrounded by a dense exudate that causes inflammatory changes in the entire wall of perforating blood vessels. Neither CT nor cerebral angiography permit the differential diagnosis between tuberculous and cysticercotic meningitis as they show similar changes in both conditions. Even necropsy examination of the brain may cause misdiagnosis if an inexpert pathologist does not recognise the scarce parasitic membranes within the leptomeningeal exudate. Cornerstones for an accurate differential diagnosis between tuberculous and cysticercotic meningitis are the neurological examination of the patient and the result from CSF analysis. Patients with NCC rarely, if ever, have fever or 
neck stiffness; likewise, the levels of CSF glucose are almost always normal in such patients. ${ }^{44}$ In contrast, patients with tuberculous meningitis usually have fever, neck stiffness, and decreased CSF glucose levels. ${ }^{12}$

The diagnosis of cysticercotic angiitis has important therapeutic implications. Those patients should be started on steroid therapy as soon as the diagnosis is established to ameliorate the subarachnoid inflammatory reaction which may cause recurrent cerebral infarcts. Further follow up with serial CSF analysis will permit, on individual basis, the tapering of steroids if the severity of inflammation has subsided. This approach is in contrast with that used in patients with atherosclerotic ischaemic stroke, where steroids have proved to be harmful. ${ }^{13}$ The diagnosis of NCC should be included in the list of causes of stroke in the young, particularly in endemic areas, and this condition must be ruled out by proper integration of data from the neurological examination, CT findings, and CSF analysis before other therapeutic measures are attempted.

Patients with meningeal NCC may develop a cerebral infarct while receiving anticysticercal drugs. Praziquantel and albendazole are highly active drugs against parenchymal brain cysticerci, ${ }^{42-44}$ and there is increasing evidence that they may also be useful in patients with meningeal cysts. ${ }^{17}$ In the latter, however, the intense inflammatory reaction developed by the host in response to the acute destruction of the parasites within the subarachnoid space, may enhance the process of angiitis in the neighbouring blood vessels favouring their occlusion. Robles ${ }^{32}$ reported a patient who died from a cerebral infarct shortly after receiving praziquantel, and we have recently described another patient with a suprasellar cysticercus who developed a cerebral infarct during a course of praziquantel. ${ }^{6}$ When treating patients with meningeal NCC, a short course of steroids seems justified to decrease such inflammatory reaction, avoiding the risk of a cerebral infarct.

1 Adams RD, Victor M. Principles of neurology, 4th Ed. New York: McGraw-Hill, 1989.

2 Alarcón T, Olivares L. Cisticercosis cerebral: manifestaciones clínicas en un medio de alta prevalencia. Rev Invest Clin (Mex) 1975;27:209-15.

3 Asbury AK, McKhann GM, McDonald W. Diseases of the nervous system. Clin

4 Saunders, 1986. . F Causas no aterosclerosas de isque mia cerebral. Arch Inst Nal Neurol Neurocir (Mex 4) mia cere $1989 ; 33$.

5 Barinagarrementeria F, Del Brutto OH. Neurocysticercosis and pure motor hemiparesis. Stroke 1988;19:1156-8.

6 Baringarrementeria F, Del Brutto $\mathrm{OH}$. Lacunar syndrome due to neurocysticercosis. Arch Neurol 1989;46:415-17.

7 Barinagarrementeria F, Del Brutto $\mathrm{OH}$, Otero E. Ataxic hemiparesis from cysticercosis. Arch Neurol 1988; 45:246.

8 Barnett HJM, Stein BM, Mohr JP, Yatsu FM. Stroke: Pathophysiology, Diagnosis, and Management. New York Churchill Livingstone, 1986.

9 Bickerstaff ER. Cerebral cysticercosis: common but unfamiliar manifestations. BMF 1955;1:1055-8

10 Botero D. Estudio sobre cisticercosis en Colombia. Rev UIS-Medicina, Bucaramanga (Col) 1986;14:19-34

11 Carbajal JR, Palacios E, Azar-Kia B, Churchill R. Radiology of cysticercosis of the central nervous system including computed tomography. Radiology 1979;125:127-31.

12 Clark WC, Metcalf JC Jr, Muhlbauer MS, Dohan FC Jr, Robertson J. Mycobacterium tuberculosis meningitis: a Robertson J. Mycobacterium tuberculosis meningitis: a
report of twelve cases and a literature review. Neurosurgery report of twelve case

13 De Reuck J, Vandekerckhove T, Bosma G, et al. Steroid treatment in acute ischemic stroke: a comparative retro- spective study of 556 tases. Eur Neurol 1988;28:70-72.

14 Del Brutto OH, Garcia E. Talamás O, Sotelo J. Sex- related severity of inflammation in parenchymal brain cysticercosis. Arch Intern Med 1988;145:544-6.

15 Del Brutto OH, Sotelo J. Neurocysticercosis: an update. Rev Infect Dis 1988;10:1075-87.

16 Del Brutto $\mathrm{OH}$, Sotelo J. Another disturbing ring. Clini Pearls 1988;11(1);7-8.

17 Del Brutto OH, Sotelo J. Albendazole therapy for subarachnoid and ventricular cysticercosis. Case report. $\mathcal{f}$ Neurosurg 1990;72:816-8.

18 Earnest MP, Reller LB, Filley CM, Grek AJ. Cysticercosis in the United States: 35 cases and a review. Rev Infect Dis 1987;9:961-79.

19 Escobar A. The pathology of neurocysticercosis. In: Palacios E, Rodriguez-Carbajal J, Taveras JM, eds. Cysticercosis of the central nervous system. Springfield: Charles C Thomas, the central nervo

20 Escobar A, Nieto D. Parasitic diseases. In: Minckler J, ed. Pathology of the nervous system, vol 3. New York: McGrawHill, 1972:2503-21.

21 García-Albea E. Cisticercosis en España: algunos datos epidemiológicos. Rev Clin Esp 1989;184:3-6.

22 Leiguarda R, Berthier M, Starkstein S, Nogües M, Lylyk P. Ischemic infarction in 25 children with tuberculous meningitis. Stroke 1988;19:200-4.

23 Loo L, Braude A. Cerebral cysticercosis in San Diego: a report of 23 cases and a review of the literature. Medicine 1982;61:341-59.

24 Lyons EL, Leeds NE. The angiographic demonstration of arterial vascular disease in purulent meningitis. Radiology 1967;88:935-8.

25 MacKenzie RA, Forbes GS, Karnes WE. Angiographic findings in herpes zoster arteritis. Ann Neurol 1981; 10:458-64.

26 McCormick GF, Giannotta S, Zee C-S, Fisher M. Carotid occlusion in cysticercosis. Neurology 1983;33:1078-80.

27 McCormick GF, Zee C-S, Heiden J. Cysticercosis cerebri: review of 127 cases. Arch Neurol 1982;39:534-9.

28 Nieto D. Cysticercosis of the nervous system: diagnosis by means of the spinal fluid complement fixation test. Neurology 1956; 6:725-38.

29 Nieto D. Historical notes on cysticercosis. In: Flisser A, Willms K, Laclete JP, Larralde C, Ridaura C, Beltrán F, eds. Cysticercosis: present state of knowledge and perspectives. New York: Academic Press, 1982:1-7.

30 Rangel R, Torres B, Del Brutto OH, Sotelo J. Cysticercotic encephalitis: a severe form in young females. $A m \mathcal{F}$ Trop Med Hyg 1987;36:387-92.

31 Richards FO Jr, Schantz PM, Ruiz-Tiben E, Sorvillo FI. Cysticercosis in Los Angeles county. $\mathcal{F} A M A$ 1985; 254:3444-8.

32 Robles C. Mortalidad en 100 enfermos con neurocisticercosis tratados con praziquantel. Salud Pub 1982; 24:629-32.

33 Rocca E, Monteagudo E. An angiographic study of neurocysticercosis. Int ₹ Surg 1966;46:130-41.

34 Rodriguez-Carbajal J, Del Brutto OH, Penagos P, Huebe J, Escobar A. Occlusion of the middle cerebral artery due to cysticercotic angiitis. Stroke 1989;20:1095-8.

35 Rosas N, Sotelo J, Nieto D. ELISA in the diagnosis of neurocysticercosis. Arch Neurol 1986;43:353-6.

36 Ross R T. Transient tumor attacks. Arch Neurol 1983; 40:633-6.

37 Saul RF, Gallagher,JG, Mateer JE. Sudden hemiparesis as the presenting sign in cryptococcal meningoencephalitis. Stroke 1986;17:753-4.

38 Scharf $D$. Neurocysticercosis: two hundred and thirty-eight cases from a California hospital. Arch Neurol 1988; 45:777-80.

39 Shanley JD, Jordan MC. Clinical aspects of CNS cysticercosis. Arch Intern Med 1980;140:1309-13.

40 Solé́lenas J Pons-Tortella E Cerebral angitis. Neuroradiology 1978;15:3-11.

41 Sotelo J. Neurocysticercosis. In: Kennedy PGE, Johnson $\mathrm{RT}$, eds. Infections of the nervous system. London: Butterworth, 1987:145-55.

42 Sotelo J, Del Brutto $\mathrm{OH}$. Therapy of neurocysticercosis. Childs Nerv Syst 1987;3:208-11.

43 Sotelo J, Del Brutto OH, Penagos P, et al. Comparison of therapeutic regimen of anticysticercal drugs for parenchymal brain cysticercosis. $\mathcal{f}$ Neurol 1990;237:69-72. Sotelo J, Escobedo F, Penagos P, Del Brutto OH. Short
course of albendazole therapy for neurocysticercosis. Arch Neurol 1988;45:1130-3.

45 Sotelo J, Guerrero V, Rubio F. Neurocysticercosis: a new classification based on active and inactive forms. Arch Intern Med 1985;145:442-5.

46 Sotelo J, Marín C. Hydrocephalus secondary to cysticercotic arachnoiditis: a long-term follow up review of 92 cases. I Neurosurg 1987;66:686-9.

47 Torrealba G, Del Villar S, Tagle P, Arriagada P, Kase CS. Cysticercosis of the central nervous system: clinical and therapeutic considerations. $\mathcal{F}$ Neurol Neurosurg Psychiatry therapeutic cons

48 Trelles JO, Trelles L. Cysticercosis of the nervous system. In: Vinken PJ, Bruyn GW, eds. Handbook of clinical
neurology, vol 35. Amsterdam: North-Holland, 1978: neurology,

49 Veenedal-Hilbers JA, Perquin WVM, Hoogland PH, Doornbos L. Basal meningo-vasculitis and occlusion of the basilar artery in two cases of Borrelia burgdorferi infection. Neurology 1988;38:1317-19.

50 Zenteno-Alanis G. Sindrome mesencefálico progresivo en cisticercosis cerebral. Presented at the XXIII Latinoamerican congress of neurosurgery, Acapulco, November 1988. 